\title{
Uniqueness of measure extensions in Banach spaces
}

\author{
by
}

\author{
J. Rodríguez and G. Vera (Murcia)
}

\begin{abstract}
Let $X$ be a Banach space, $B \subset B_{X^{*}}$ a norming set and $\mathfrak{T}(X, B)$ the topology on $X$ of pointwise convergence on $B$. We study the following question: given two (non-negative, countably additive and finite) measures $\mu_{1}$ and $\mu_{2}$ on $\operatorname{Baire}(X, w)$ which coincide on $\operatorname{Baire}(X, \mathfrak{T}(X, B))$, does it follow that $\mu_{1}=\mu_{2}$ ? It turns out that this is not true in general, although the answer is affirmative provided that both $\mu_{1}$ and $\mu_{2}$ are convexly $\tau$-additive (e.g. when $X$ has the Pettis Integral Property). For a Banach space $Y$ not containing isomorphic copies of $\ell^{1}$, we show that $Y^{*}$ has the Pettis Integral Property if and only if every measure on Baire $\left(Y^{*}, w^{*}\right)$ admits a unique extension to $\operatorname{Baire}\left(Y^{*}, w\right)$. We also discuss the coincidence of the two $\sigma$-algebras involved in such results. Some other applications are given.
\end{abstract}

1. Introduction. All the measures considered in this paper are nonnegative, countably additive and finite. A basic question in measure theory is the following: Given two $\sigma$-algebras $\Sigma^{\prime} \subset \Sigma$ on a set $\Omega$ and two measures $\mu_{1}$ and $\mu_{2}$ on $\Sigma$ such that $\left.\mu_{1}\right|_{\Sigma^{\prime}}=\left.\mu_{2}\right|_{\Sigma^{\prime}}$, when does it follow that $\mu_{1}=\mu_{2}$ ? The purpose of this paper is to discuss this problem in the setting of Baire $\sigma$-algebras of weak topologies in Banach spaces. More precisely, we are concerned with

$$
\Sigma^{\prime}=\operatorname{Baire}(X, \mathfrak{T}(X, B)) \subset \Sigma=\operatorname{Baire}(X, w),
$$

where $X$ is a Banach space, $B \subset B_{X^{*}}$ is a norming set and $\mathfrak{T}(X, B)$ is the topology on $X$ of pointwise convergence on $B$, which is weaker than the weak topology $w=\mathfrak{T}\left(X, B_{X^{*}}\right)$ (for all unexplained notation and terminology, we refer the reader to the end of this section). We emphasize that the Baire $\sigma$ algebra of a locally convex space endowed with its weak topology is exactly the $\sigma$-algebra generated by all the elements of the topological dual [7]. In particular, Baire $(X, \mathfrak{T}(X, B))$ is the $\sigma$-algebra on $X$ generated by $B$.

2000 Mathematics Subject Classification: 28A20, 28B05, 28C15, 46B26, $46 \mathrm{G} 10$.

Key words and phrases: measure extension, Baire measure, Banach space, Pettis integral.

This research was partially supported by the grants BFM2002-01719 of MCyT (Spain) and 00690/PI/04 of Fundación Séneca (CARM, Spain). The first named author was also partially supported by a FPU grant of MEC (Spain). 
We next summarize the content of this paper. Section 2 plays an auxiliary role and is devoted to providing, in a general measure-theoretic setting, a criterion for the uniqueness of measure extensions (Theorem 2.7) which can be applied successfully in Section 3 within the framework of weak Baire measures in Banach spaces. Our approach relies on Edgar's work [9] (going back to [17]) about the continuity of the integral over a uniformly integrable set of functions endowed with the pointwise convergence topology.

In Section 3 our main results are proved. It turns out that there is a close relationship between the theory of the Pettis integral and the problems considered in this work. Following [22], we say that a measure $\mu$ on $\operatorname{Baire}(X, w)$ is convexly $\tau$-additive if for each decreasing net $\left(C_{\alpha}\right)$ of convex closed elements of $\operatorname{Baire}(X, w)$ with $\bigcap_{\alpha} C_{\alpha}=\emptyset$, we have $\lim _{\alpha} \mu\left(C_{\alpha}\right)=0$. It is well known that a scalarly bounded function $f$ defined on a measure space $(\Lambda, \mathcal{S}, \nu)$ with values in $X$ is Pettis integrable if and only if the image measure $\nu f^{-1}$ on $\operatorname{Baire}(X, w)$ is convexly $\tau$-additive [26, 5-2-4]. Thus, $X$ has the Pettis Integral Property $(P I P)$ if and only if every measure on $\operatorname{Baire}(X, w)$ is convexly $\tau$-additive (Remark 3.3). As an immediate application of the results proved in Section 2, we show that the question raised in the first paragraph has affirmative answer for $\Sigma^{\prime}=\operatorname{Baire}(X, \mathfrak{T}(X, B)) \subset \Sigma=\operatorname{Baire}(X, w)$ provided that both $\mu_{1}$ and $\mu_{2}$ are convexly $\tau$-additive (Theorem 3.2).

Of course, the previous result is not of interest when

$$
\operatorname{Baire}(X, \mathfrak{T}(X, B))=\operatorname{Baire}(X, w) .
$$

Remember that this equality holds, for instance, whenever $\left(B_{X^{*}}\right.$, weak $\left.{ }^{*}\right)$ is angelic. In Subsection 3.1 we study the coincidence of both $\sigma$-algebras, including examples of Banach spaces with the PIP for which Baire $(X, \mathfrak{T}(X, B))$ $\neq \operatorname{Baire}(X, w)$. Our attention is mainly focused on the case in which $X=Y^{*}$ and $B=B_{Y}$, where $Y$ is a Banach space. (Note that $w^{*}=\mathfrak{T}\left(Y^{*}, B\right)$ is just the weak* topology on $Y^{*}$.) We prove that $\operatorname{Baire}\left(Y^{*}, w^{*}\right)=\operatorname{Baire}\left(Y^{*}, w\right)$ if and only if $Y$ is sequentially dense in $\left(Y^{* *}\right.$, weak $\left.{ }^{*}\right)$ (Proposition 3.9) and that these conditions are satisfied whenever $Y^{*}$ has property (C) (Corollary 3.10).

The uniqueness of measure extensions in the setting of dual Banach spaces is analyzed in Subsection 3.2. We consider the following class of spaces.

Definition 1.1. Let $Y$ be a Banach space. We say that $Y^{*}$ has the Uniqueness of Measure Extensions Property (UMEP for short) if for every pair of measures $\mu_{1}$ and $\mu_{2}$ on $\operatorname{Baire}\left(Y^{*}, w\right)$ we have

$$
\left.\mu_{1}\right|_{\operatorname{Baire}\left(Y^{*}, w^{*}\right)}=\left.\mu_{2}\right|_{\operatorname{Baire}\left(Y^{*}, w^{*}\right)} \Rightarrow \mu_{1}=\mu_{2} .
$$

In view of the comments above, every dual Banach space $Y^{*}$ with the PIP has the UMEP. Furthermore, our Theorem 3.17 states that the converse holds when $Y$ does not contain subspaces isomorphic to $\ell^{1}$. We also show that in general every dual Banach space with the UMEP is realcompact for its 
weak topology (Proposition 3.18). The converse is not true in general, since $\ell_{\infty}$ fails the UMEP (Example 3.19).

Notation and terminology. For all unexplained terminology and notation we refer the reader to the standard references [11] (Banach spaces), [13] (topological measure theory) and [26] (Pettis integral).

Given a set $\Omega$, we write $\mathfrak{T}_{p}(\Omega)$ (or simply $\mathfrak{T}_{p}$ ) to denote the topology on $\mathbb{R}^{\Omega}$ of pointwise convergence on $\Omega$. We denote by $\sigma(\mathcal{F})$ the $\sigma$-algebra on $\Omega$ generated by a family $\mathcal{F} \subset \mathbb{R}^{\Omega}$ (i.e. the smallest one for which each element of $\mathcal{F}$ is measurable). As usual, $\operatorname{co}(\mathcal{F})$ (resp. aco $(\mathcal{F})$ ) stands for the convex (resp. absolutely convex) hull of $\mathcal{F}$ in $\mathbb{R}^{\Omega}$.

Let $(T, \mathfrak{T})$ be a completely regular Hausdorff topological space. We denote by $\operatorname{Baire}(T, \mathfrak{T})$ (resp. $\operatorname{Borel}(T, \mathfrak{T}))$ the $\sigma$-algebra on $T$ generated by the family of all real-valued continuous functions on $T$ (resp. closed subsets of $T)$. We write $\mathcal{M}_{\sigma}(T, \mathfrak{T})$ to denote the family of all measures on $\operatorname{Baire}(T, \mathfrak{T})$. We say that $\mu \in \mathcal{M}_{\sigma}(T, \mathfrak{T})$ is tight if $\mu(T)=\sup \left\{\mu^{*}(K)\right.$ : $K \subset T, K$ is compact $\}$, where $\mu^{*}$ stands for the outer measure induced by $\mu$. A measure $\nu$ on $\operatorname{Borel}(T, \mathfrak{T})$ is called a Radon measure if $\nu(E)=\sup \{\nu(K)$ : $K \subset E, K$ is compact $\}$ for every $E \in \operatorname{Borel}(T, \mathfrak{T})$. It is well known that every tight measure on $\operatorname{Baire}(T, \mathfrak{T})$ can be extended (in a unique way) to a Radon measure on $\operatorname{Borel}(T, \mathfrak{T})$. The $\sigma$-algebra made up of all universally measurable subsets of $T$ (i.e. those which are measurable with respect to each Radon measure on $\operatorname{Borel}(T, \mathfrak{T}))$ will be denoted by $\operatorname{Univ}(T, \mathfrak{T})$. Recall that $(T, \mathfrak{T})$ is called angelic if each relatively countably compact set $A \subset T$ is relatively compact and for every $t \in \bar{A}$ there is a sequence in $A$ converging to $t$.

All our Banach spaces $Z$ are assumed to be real. We write $B_{Z}$ to denote the closed unit ball of $Z$. As usual, $Z^{*}$ stands for the topological dual of $Z$. Given $z \in Z$ and $z^{*} \in Z^{*}$, we sometimes write $\left\langle z^{*}, z\right\rangle$ instead of $z^{*}(z)$. We identify $Z$ as a closed subspace of $Z^{* *}$ by means of the canonical isometry. A set $B \subset B_{Z^{*}}$ is said to be norming if $\|z\|=\sup \left\{\left|z^{*}(z)\right|: z^{*} \in B\right\}$ for every $z \in Z$. The topology $\mathfrak{T}(Z, B)$ is the coarsest one for which each element of $B$ is continuous. We write $Z \not \supset \ell^{1}$ if $Z$ does not contain subspaces isomorphic to $\ell^{1}$. Recall that $Z$ has property (C) (introduced by Corson [3]) if every family of closed convex subsets of $Z$ with empty intersection contains a countable subfamily with empty intersection.

Given a measure space $(\Omega, \Sigma, \mu)$, we write $\mathcal{L}^{1}(\mu)$ to denote the space of all $\Sigma$-measurable and $\mu$-integrable real-valued functions defined on $\Omega$, and $L^{1}(\mu)$ for the corresponding Banach space of equivalence classes with its usual norm $\|\cdot\|_{1}$. A set $\mathcal{H} \subset \mathcal{L}^{1}(\mu)$ is uniformly integrable if it is $\|\cdot\|_{1^{-}}$ bounded and for each $\varepsilon>0$ there is $\delta>0$ such that $\sup _{h \in \mathcal{H}} \int_{E}|h| d \mu \leq \varepsilon$ whenever $\mu(E) \leq \delta$. A function $f: \Omega \rightarrow Z$ is said to be 
(i) scalarly measurable if the composition $\left\langle z^{*}, f\right\rangle$ is $\Sigma$-measurable for every $z^{*} \in Z^{*}$

(ii) scalarly bounded if it is scalarly measurable and there is $M>0$ such that for every $z^{*} \in B_{Z^{*}}$ we have $\left|\left\langle z^{*}, f\right\rangle\right| \leq M \mu$-a.e.;

(iii) Pettis integrable if $\left\langle z^{*}, f\right\rangle \in \mathcal{L}^{1}(\mu)$ for every $z^{*} \in Z^{*}$ and for each $E \in \Sigma$ there is $z_{E} \in Z$ such that $\int_{E}\left\langle z^{*}, f\right\rangle d \mu=z^{*}\left(z_{E}\right)$ for every $z^{*} \in Z^{*}$.

We say that $Z$ has the $\mu$-PIP if each scalarly bounded function from $\Omega$ to $Z$ is Pettis integrable. The space $Z$ has the Pettis Integral Property if it has the $\mu$-PIP for every measure space $(\Omega, \Sigma, \mu)$. It is known that

$$
\left(B_{Z^{*}} \text {, weak }{ }^{*}\right) \text { angelic } \Rightarrow Z \text { has property }(\mathrm{C}) \Rightarrow Z \text { has the PIP, }
$$

and none of the reverse implications holds in general (see $[8,23,26])$.

2. A criterion for the uniqueness of measure extensions. In order to deal with Theorem 2.7 we need some preliminary work, which we have divided into a sequence of lemmas for the convenience of the reader.

DeFinition 2.1. Let $\Sigma^{\prime} \subset \Sigma$ be two $\sigma$-algebras on a set $\Omega$.

(1) Let $\mathcal{M}$ be a family of measures on $\Sigma$. We say that $\Sigma^{\prime}$ has the uniqueness property with respect to $\mathcal{M}$ if for every pair $\mu_{1}, \mu_{2} \in \mathcal{M}$ we have

$$
\left.\mu_{1}\right|_{\Sigma^{\prime}}=\left.\mu_{2}\right|_{\Sigma^{\prime}} \Rightarrow \mu_{1}=\mu_{2} .
$$

(2) We say that a measure $\mu$ on $\Sigma$ is approximated by $\Sigma^{\prime}$ if for every $E \in \Sigma$ there is $B \in \Sigma^{\prime}$ such that $\mu(E \triangle B)=0$.

Lemma 2.2. Let $\Sigma^{\prime} \subset \Sigma$ be two $\sigma$-algebras on a set $\Omega$, and $\mathcal{M}$ a family of measures on $\Sigma$ such that $\mu_{1}+\mu_{2} \in \mathcal{M}$ for every $\mu_{1}, \mu_{2} \in \mathcal{M}$. Suppose that every element of $\mathcal{M}$ is approximated by $\Sigma^{\prime}$. Then $\Sigma^{\prime}$ has the uniqueness property with respect to $\mathcal{M}$.

Proof. Fix $\mu_{1}, \mu_{2} \in \mathcal{M}$ such that $\left.\mu_{1}\right|_{\Sigma^{\prime}}=\left.\mu_{2}\right|_{\Sigma^{\prime}}$. Since $\mu:=\mu_{1}+\mu_{2}$ belongs to $\mathcal{M}$, for each $E \in \Sigma$ there is $B \in \Sigma^{\prime}$ such that $\mu(E \triangle B)=0$, hence $\mu_{1}(E \triangle B)=\mu_{2}(E \triangle B)=0$ and therefore $\mu_{1}(E)=\mu_{1}(B)=\mu_{2}(B)=$ $\mu_{2}(E)$.

It is worth pointing out that the converse of Lemma 2.2 holds true under certain additional assumptions on the family of measures, as we show in Corollary 2.3 below. Given two $\sigma$-algebras $\Sigma^{\prime} \subset \Sigma$ on a set $\Omega$ and a measure $\mu^{\prime}$ on $\Sigma^{\prime}$, we write ca $\left(\mu^{\prime}, \Sigma\right)$ for the convex set of all measures $\mu$ on $\Sigma$ such that $\left.\mu\right|_{\Sigma^{\prime}}=\mu^{\prime}$. A well known result of R. G. Douglas (see [4, 21]) states that a measure $\mu \in \operatorname{ca}\left(\mu^{\prime}, \Sigma\right)$ is an extreme point of $\operatorname{ca}\left(\mu^{\prime}, \Sigma\right)$ if and only if $\mu$ is approximated by $\Sigma^{\prime}$. 
Corollary 2.3. Let $\Sigma^{\prime} \subset \Sigma$ be two $\sigma$-algebras on a set $\Omega$ and $\mathcal{M}$ a family of measures on $\Sigma$ with the following properties:

- $\mu_{1}+\mu_{2} \in \mathcal{M}$ for every $\mu_{1}, \mu_{2} \in \mathcal{M}$;

- if $\nu \in \mathcal{M}$ and $\mu$ is a measure on $\Sigma$ such that $\mu \leq \nu$, then $\mu \in \mathcal{M}$.

Then $\Sigma^{\prime}$ has the uniqueness property with respect to $\mathcal{M}$ if and only if every element of $\mathcal{M}$ is approximated by $\Sigma^{\prime}$.

Proof. It only remains to show the only if part. So assume that $\Sigma^{\prime}$ has the uniqueness property with respect to $\mathcal{M}$ and fix $\mu \in \mathcal{M}$. We claim that $\mu$ is an extreme point of $\mathrm{ca}\left(\left.\mu\right|_{\Sigma^{\prime}}, \Sigma\right)$. Indeed, write $\mu=\left(\mu_{1}+\mu_{2}\right) / 2$, where $\mu_{1}, \mu_{2} \in \operatorname{ca}\left(\left.\mu\right|_{\Sigma^{\prime}}, \Sigma\right)$. Since $\mu_{i} \leq \mu_{1}+\mu_{2}=\mu+\mu \in \mathcal{M}$ for $i=1$, 2, we infer that $\mu_{1}, \mu_{2} \in \mathcal{M}$ and therefore $\mu_{1}=\mu_{2}$. This proves the claim. An appeal to Douglas' aforementioned result now ensures that $\mu$ is approximated by $\Sigma^{\prime}$.

The following lemma provides a useful sufficient condition for a measure to be approximated by a sub- $\sigma$-algebra.

LEMmA 2.4. Let $\Omega$ be a set, $\mathcal{F}^{\prime} \subset \mathcal{F} \subset \mathbb{R}^{\Omega}$ two families, and $\mu$ a measure on $\sigma(\mathcal{F})$. Suppose that for each $f \in \mathcal{F}$ there is a sequence $\left(f_{n}\right)$ in $\mathcal{F}^{\prime}$ that converges to $f \mu$-a.e. Then $\mu$ is approximated by $\sigma\left(\mathcal{F}^{\prime}\right)$.

Proof. It is easy to check that the family

$$
\mathcal{A}=\left\{E \in \sigma(\mathcal{F}): \text { there is } B \in \sigma\left(\mathcal{F}^{\prime}\right) \text { such that } \mu(E \triangle B)=0\right\}
$$

is a $\sigma$-algebra on $\Omega$ contained in $\sigma(\mathcal{F})$. Therefore, in order to show that $\mathcal{A}=\sigma(\mathcal{F})$ it suffices to prove that each $f \in \mathcal{F}$ is $\mathcal{A}$-measurable. To this end, fix $f \in \mathcal{F}, t \in \mathbb{R}$ and define $H:=\{\omega \in \Omega: f(\omega)>t\}$. By the assumption, there is a sequence $\left(f_{n}\right)$ in $\mathcal{F}^{\prime}$ that converges to $f \mu$-a.e. Fix $F \in \Sigma$ such that $\mu(\Omega \backslash F)=0$ and $\lim _{n} f_{n}(\omega)=f(\omega)$ for every $\omega \in F$. The set

$$
B:=\bigcup_{n=1}^{\infty} \bigcup_{m=1}^{\infty} \bigcap_{k \geq m}\left\{\omega \in \Omega: f_{k}(\omega)>t+1 / n\right\}
$$

belongs to $\sigma\left(\mathcal{F}^{\prime}\right)$ and satisfies $B \cap F=H \cap F$, hence $\mu(H \triangle B)=0$ and therefore $H \in \mathcal{A}$. As $t \in \mathbb{R}$ is arbitrary, $f$ is $\mathcal{A}$-measurable. The proof is finished.

The notion of $\mathcal{F}$-smooth measure defined below includes as particular cases the convexly $\tau$-additive weak Baire measures on Banach spaces (see Lemma 3.1 in Section 3) as well as the $\tau$-additive Baire measures on completely regular Hausdorff topological spaces.

Definition 2.5. Let $(\Omega, \Sigma)$ be a measurable space and $\mathcal{F} \subset \mathbb{R}^{\Omega}$ a family of $\Sigma$-measurable functions. Denote by $\mathcal{Z}_{\mathcal{F}}$ the collection of all finite intersections of sets of the form $\{\omega \in \Omega: f(\omega) \leq g(\omega)+t\}$, where $f, g \in \mathcal{F}$ and 
$t \in \mathbb{R}$. A measure $\mu$ on $\Sigma$ is said to be $\mathcal{F}$-smooth if for each decreasing net $\left(Z_{\alpha}\right)$ of elements of $\mathcal{Z}_{\mathcal{F}}$ with $\bigcap_{\alpha} Z_{\alpha}=\emptyset$, we have $\lim _{\alpha} \mu\left(Z_{\alpha}\right)=0$.

Our interest in considering $\mathcal{F}$-smooth measures is motivated by the "separation property" isolated in Lemma 2.6, which will allow us to use a result of Edgar [9] regarding the continuity of the "identity" mapping

$$
I:\left(\mathcal{F}, \mathfrak{T}_{p}\right) \rightarrow\left(L^{1}(\mu), \text { weak }\right)
$$

(that sends each function to its equivalence class) when $\mathcal{F}$ is a $\mathfrak{T}_{p^{-}}$countably compact, convex and uniformly integrable subset of $\mathcal{L}^{1}(\mu)$.

Lemma 2.6. Let $(\Omega, \Sigma)$ be a measurable space, $\mathcal{F} \subset \mathbb{R}^{\Omega}$ a family of $\Sigma$-measurable functions and $\mu$ an $\mathcal{F}$-smooth measure on $\Sigma$. Let $S:=$ $\bigcap\left\{Z \in \mathcal{Z}_{\mathcal{F}}: \mu(\Omega \backslash Z)=0\right\}$. Then for every pair $f, g \in \mathcal{F}$ we have

$$
\left.f\right|_{S}=\left.g\right|_{S} \Leftrightarrow f=g \mu \text {-a.e. }
$$

Proof. Notice that the family $\mathcal{C}:=\left\{Z \in \mathcal{Z}_{\mathcal{F}}: \mu(\Omega \backslash Z)=0\right\}$ is nonempty and closed under finite intersections. Fix $f, g \in \mathcal{F}$. If $f=g \mu$-a.e., then $Z:=\{\omega \in \Omega: f(\omega)=g(\omega)\} \in \mathcal{C}$, so $S \subset Z$ and therefore $\left.f\right|_{S}=\left.g\right|_{S}$. Conversely, suppose that $\left.f\right|_{S}=\left.g\right|_{S}$ and fix $n \in \mathbb{N}$. Define

$$
C_{n}:=\{\omega \in \Omega: f(\omega) \geq g(\omega)+1 / n\} \in \mathcal{Z}_{\mathcal{F}} .
$$

Since the family $\mathcal{C}_{n}:=\left\{Z \cap C_{n}: Z \in \mathcal{C}\right\} \subset \mathcal{Z}_{\mathcal{F}}$ is closed under finite intersections and $\bigcap \mathcal{C}_{n}=S \cap C_{n}=\emptyset$, we can apply the $\mathcal{F}$-smoothness of $\mu$ to deduce that $\mu\left(C_{n}\right)=\inf \left\{\mu\left(Z \cap C_{n}\right): Z \in \mathcal{C}\right\}=0$. As $n \in \mathbb{N}$ is arbitrary, $f \leq g \mu$-a.e. A similar argument yields $f \geq g \mu$-a.e., completing the proof.

Given a measure space $(\Omega, \Sigma, \mu)$ and $A \in \Sigma$, we write $\mu_{A}$ to denote the measure on $\Sigma$ defined by $\mu_{A}(E):=\mu(E \cap A)$.

ThEOREM 2.7. Let $\Omega$ be a set and $\mathcal{F}^{\prime} \subset \mathcal{F} \subset \mathbb{R}^{\Omega}$ two families of functions such that

- $\mathcal{F}$ is convex and $\mathfrak{T}_{p}$-countably compact;

- $\mathcal{F}^{\prime}$ is $\mathfrak{T}_{p}$-dense in $\mathcal{F}$.

Then $\sigma\left(\mathcal{F}^{\prime}\right)$ has the uniqueness property with respect to the family of all $\mathcal{F}$-smooth measures on $\sigma(\mathcal{F})$.

Proof. Since the sum of any two $\mathcal{F}$-smooth measures on $\sigma(\mathcal{F})$ is again $\mathcal{F}$-smooth, Lemmas 2.2 and 2.4 say that in order to prove the result it suffices to check that each $\mathcal{F}$-smooth measure $\mu$ on $\sigma(\mathcal{F})$ satisfies the following condition: for every $f \in \mathcal{F}$ there is a sequence $\left(f_{n}\right)$ in $\mathcal{F}^{\prime}$ that converges to $f$ $\mu$-a.e. We divide the proof into two steps.

STEP 1. Suppose that $\mathcal{F}$ is a uniformly integrable subset of $\mathcal{L}^{1}(\mu)$. Since $\mu$ is $\mathcal{F}$-smooth, Lemma 2.6 ensures that there is a set $S \subset \Omega$ such that for 
every $f, g \in \mathcal{F}$ we have

$$
\left.f\right|_{S}=\left.g\right|_{S} \Leftrightarrow f=g \mu \text {-a.e. }
$$

By the assumption, $\mathcal{F}$ is convex and $\mathfrak{T}_{p}$-countably compact, so we can apply [9, Proposition 3] to deduce that the "identity" mapping

$$
I:\left(\mathcal{F}, \mathfrak{T}_{p}\right) \rightarrow\left(L^{1}(\mu), \text { weak }\right)
$$

(that sends each function to its equivalence class) is continuous. Since $\mathcal{F}$ is convex, $\mathcal{F}^{\prime} \subset \operatorname{co}\left(\mathcal{F}^{\prime}\right) \subset \mathcal{F}$ and we can suppose without loss of generality that $\mathcal{F}^{\prime}$ is convex as well. Bearing in mind that $\mathcal{F}^{\prime}$ is $\mathfrak{T}_{p}$-dense in $\mathcal{F}$, the continuity of $I$ yields

$$
I(\mathcal{F}) \subset{\overline{I\left(\mathcal{F}^{\prime}\right)}}^{\text {weak }}=\overline{I\left(\mathcal{F}^{\prime}\right)} \|^{\|\cdot\|_{1}} .
$$

It follows that for each $f \in \mathcal{F}$ there is a sequence $\left(f_{n}\right)$ in $\mathcal{F}^{\prime}$ such that $\lim _{n}\left\|f_{n}-f\right\|_{1}=0$, and thus we can find a subsequence $\left(f_{n_{k}}\right)$ converging to $f \mu$-a.e.

STEP 2. Since $\mathcal{F}$ is a pointwise bounded (because it is $\mathfrak{T}_{p}$-countably compact) family of $\sigma(\mathcal{F})$-measurable functions, there is a non-negative $\sigma(\mathcal{F})$ measurable function $h \in \mathbb{R}^{\Omega}$ such that for each $f \in \mathcal{F}$ we have $|f| \leq h \mu$-a.e. (see e.g. $[10,4.1 .1])$. Therefore, we can find a sequence $\left(A_{k}\right)_{k=1}^{\infty}$ in $\sigma(\mathcal{F})$ such that for every $k \in \mathbb{N}$,

- $\mathcal{F}$ is a uniformly integrable subset of $\mathcal{L}^{1}\left(\mu_{A_{k}}\right)$;

- $\mu\left(\Omega \backslash A_{k}\right) \leq 1 / 2^{k}$.

Given $k \in \mathbb{N}$, in view of Step 1 (notice that $\mu_{A_{k}}$ is $\mathcal{F}$-smooth) there is a sequence $\left(f_{n}^{k}\right)_{n=1}^{\infty}$ in $\mathcal{F}^{\prime}$ converging to $f \mu_{A_{k}}$-a.e. By Egorov's theorem, there exist $E_{k} \in \sigma(\mathcal{F})$ with $\mu_{A_{k}}\left(\Omega \backslash E_{k}\right) \leq 1 / 2^{k}$ and $n(k) \in \mathbb{N}$ such that $\sup _{\omega \in E_{k}}\left|f_{n(k)}^{k}(\omega)-f(\omega)\right| \leq 1 / k$. Fix $s \in \mathbb{N}$. We have

$$
N_{s}:=\bigcap_{l=1}^{\infty} \bigcup_{k=l}^{\infty}\left\{\omega \in \Omega:\left|f_{n(k)}^{k}(\omega)-f(\omega)\right|>1 / s\right\} \subset \bigcap_{l=s}^{\infty} \bigcup_{k=l}^{\infty}\left(\Omega \backslash E_{k}\right),
$$

hence $N_{s} \in \sigma(\mathcal{F})$ satisfies

$\mu\left(N_{s}\right) \leq \sum_{k=l}^{\infty} \mu\left(\Omega \backslash E_{k}\right) \leq \sum_{k=l}^{\infty} \mu_{A_{k}}\left(\Omega \backslash E_{k}\right)+\sum_{k=l}^{\infty} \mu\left(\Omega \backslash A_{k}\right) \leq \sum_{k=l}^{\infty} \frac{1}{2^{k-1}}=2^{-l+2}$

for every $l \geq s$ and therefore $\mu\left(N_{s}\right)=0$. Finally, notice that $\left(f_{n(k)}^{k}\right)_{k=1}^{\infty}$ converges to $f$ pointwise on $\Omega \backslash \bigcup_{s=1}^{\infty} N_{s}$, and so $\mu$-a.e. The proof is complete.

We mention that some other known facts concerning the continuity of the "identity" mapping $J:\left(\mathcal{F}, \mathfrak{T}_{p}\right) \rightarrow\left(L^{1}(\mu),\|\cdot\|_{1}\right)$ (see [9] and the survey paper [28] for a thorough study on this subject) could be applied in a similar way to deduce results along the line of Theorem 2.7 without the assumption of convexity on $\mathcal{F}$. 
3. Uniqueness of extensions in Banach spaces. Theorem 3.2 below is now an easy consequence of Theorem 2.7. We first need to recall the following well known fact which we do not find in print.

Lemma 3.1. Let $X$ be a Banach space and $\mu \in \mathcal{M}_{\sigma}(X, w)$. Then $\mu$ is

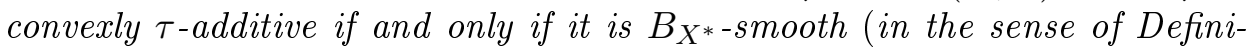
tion 2.5).

Proof. Since $\mathcal{Z}_{B_{X^{*}}}$ is made up of closed convex elements of $\operatorname{Baire}(X, w)$, we only have to check the if part. To this end, we begin with the following claim.

Claim. Let $C \subset X$ be closed and convex. Then there is $\mathcal{D}_{C} \subset \mathcal{Z}_{B_{X^{*}}}$ such that $C=\bigcap \mathcal{D}_{C}$.

Indeed, let us consider the family $\mathcal{D}_{C}$ of all elements of $\mathcal{Z}_{B_{X^{*}}}$ of the form $\left\{x \in X: x^{*}(x) \leq \sup \left(x^{*}(C)\right)\right\}$, where $x^{*} \in B_{X^{*}}$ and $\sup \left(x^{*}(C)\right)<\infty$. The Hahn-Banach separation theorem ensures that $C=\bigcap \mathcal{D}_{C}$ and the Claim is proved.

Assume now that $\mu$ is $B_{X^{*-S m o o t h}}$ and fix a decreasing net $\left(C_{\alpha}\right)$ of convex closed elements of Baire $(X, w)$ with $\bigcap_{\alpha} C_{\alpha}=\emptyset$. By the Claim above, for each $\alpha$ we can find $\mathcal{D}_{\alpha} \subset \mathcal{Z}_{B_{X^{*}}}$ such that $C_{\alpha}=\bigcap \mathcal{D}_{\alpha}$. Consider now the family $\mathcal{Z} \subset \mathcal{Z}_{B_{X^{*}}}$ of all finite intersections of elements of $\bigcup_{\alpha} \mathcal{D}_{\alpha}$. Since $\mathcal{Z}$ is closed under finite intersections and $\bigcap \mathcal{Z}=\bigcap_{\alpha}\left(\bigcap_{\mathcal{D}}\right)=\bigcap_{\alpha} C_{\alpha}=\emptyset$, we

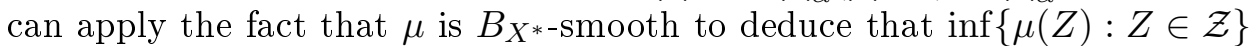
$=0$. In order to finish the proof notice that, given $Z \in \mathcal{Z}$, there exist $\alpha_{1}, \ldots, \alpha_{n}$ and $D_{i} \in \mathcal{D}_{\alpha_{i}}$ such that $Z=\bigcap_{i=1}^{n} D_{i} \supset \bigcap_{i=1}^{n} C_{\alpha_{i}}$. Since $\left(C_{\alpha}\right)$ is decreasing, there is some $\alpha$ such that $C_{\alpha} \subset \bigcap_{i=1}^{n} C_{\alpha_{i}} \subset Z$. It follows that $\lim _{\alpha} \mu\left(C_{\alpha}\right)=0$ and the proof is complete.

TheOREM 3.2. Let $X$ be a Banach space and $B \subset B_{X^{*}}$ a norming set. Then Baire $(X, \mathfrak{T}(X, B))$ has the uniqueness property with respect to the family of all convexly $\tau$-additive measures on $\operatorname{Baire}(X, w)$.

Proof. Since $B$ is norming, the Hahn-Banach separation theorem ensures that $\operatorname{aco}(B)$ is weak ${ }^{*}$-dense in $B_{X^{*}}$, that is, $\operatorname{aco}(B)$ is a $\mathfrak{T}_{p}(X)$-dense subset of the family $B_{X^{*}} \subset \mathbb{R}^{X}$. Bearing in mind that $B_{X^{*}}$ is convex and $\mathfrak{T}_{p}(X)$ compact (by Alaoglu's theorem), we can apply Theorem 2.7 to conclude that $\sigma(\operatorname{aco}(B))=\operatorname{Baire}(X, \mathfrak{T}(X, B))$ has the uniqueness property with respect to

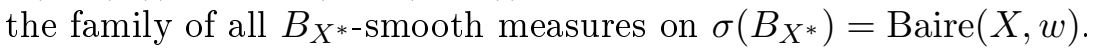

REMARK 3.3. A Banach space $X$ has the PIP if and only if every measure on $\operatorname{Baire}(X, w)$ is convexly $\tau$-additive. Indeed, this is a consequence of the fact that a scalarly bounded $X$-valued function $f$ is Pettis integrable if and only if the image measure induced by $f$ on $\operatorname{Baire}(X, w)$ is convexly $\tau$-additive (see $[26,5-2-4])$. Notice that, given a measure $\mu$ on $\operatorname{Baire}(X, w)$, there is a 
non-decreasing sequence $\left(A_{n}\right)$ in $\operatorname{Baire}(X, w)$ with union $X$ such that the identity function $I: X \rightarrow X$ is scalarly bounded with respect to each $\mu_{A_{n}}$ (see Step 2 of the proof of Theorem 2.7). Thus $\mu$ is convexly $\tau$-additive if and only if $I$ is Pettis integrable with respect to each $\mu_{A_{n}}$.

The previous remark and Theorem 3.2 have the following

Corollary 3.4. Let $X$ be a Banach space with the PIP and $B \subset B_{X^{*}}$ a norming set. Then $\operatorname{Baire}(X, \mathfrak{T}(X, B))$ has the uniqueness property with respect to $\mathcal{M}_{\sigma}(X, w)$.

3.1. Coincidence of $\operatorname{Baire}(X, \mathfrak{T}(X, B))$ and $\operatorname{Baire}(X, w)$. It is clear that, for a Banach space $X$ such that $\left(B_{X^{*}}\right.$, weak $\left.{ }^{*}\right)$ is angelic, the equality

$$
\operatorname{Baire}(X, \mathfrak{T}(X, B))=\operatorname{Baire}(X, w)
$$

holds for any norming set $B \subset B_{X^{*}}$ (since $B_{X^{*}}=\overline{\operatorname{aco}(B)}^{\text {weak* }}$ ). In fact, the same conclusion can be obtained if we only assume that $B$ separates the points of $X$ (see [15]). In particular, for this class of Banach spaces (which contains all weakly compactly generated and, more generally, all weakly Lindelöf determined spaces, see e.g. [11, Chapters 7 and 8]) the result isolated in Corollary 3.4 is futile.

In this subsection we discuss the coincidence of $\operatorname{Baire}(X, \mathfrak{T}(X, B))$ and $\operatorname{Baire}(X, w)$ in some particular cases of special interest. We will need the following lemma, which might be folklore and is included here for the convenience of the reader.

Lemma 3.5. Let $X$ be a Banach space and $F$ a subset of $X^{*}$. If $x^{*} \in X^{*}$ is $\sigma(F)$-measurable, then there is a countable set $C \subset F$ such that

$$
x^{*} \in \overline{\operatorname{span}(C)}^{\text {weak* }^{*}} .
$$

Proof. Since $x^{*}$ is $\sigma(F)$-measurable, there is a countable set $C \subset F$ such that $x^{*}$ is $\sigma(C)$-measurable. Let $\Sigma$ be the family of all elements $A \in \sigma(C)$ such that

$$
\bigcap_{y^{*} \in C} \operatorname{ker} y^{*} \subset \begin{cases}A & \text { if } 0 \in A \\ X \backslash A & \text { if } 0 \notin A\end{cases}
$$

It is easy to check that $\Sigma$ is a $\sigma$-algebra on $X$ for which each $y^{*} \in C$ is $\Sigma$-measurable, hence $\Sigma=\sigma(C)$. It follows that $\operatorname{ker} x^{*} \in \Sigma$ and therefore $\bigcap_{y^{*} \in C}$ ker $y^{*} \subset \operatorname{ker} x^{*}$. From the last inclusion and the Hahn-Banach separation theorem we infer that $x^{*}$ belongs to the weak ${ }^{*}$-closure of $\operatorname{span}(C)$. The proof is complete.

Given a compact Hausdorff topological space $K$, we write $C(K)$ to denote the Banach space of all real-valued continuous functions on $K$ endowed with the supremum norm. Note that the set $B=\left\{\delta_{t}: t \in K\right\} \subset B_{C(K)^{*}}$ of "point masses" (i.e. $\left.\delta_{t}(h):=h(t)\right)$ is norming and that $\mathfrak{T}(C(K), B)=\mathfrak{T}_{p}(K)$. We 
next study the Baire $\left(C(K), \mathfrak{T}_{p}(K)\right)$-measurability of the "integral" functional $\iota(\mu) \in C(K)^{*}, \iota(\mu)(f):=\int_{K} f d \mu$, associated to a Radon measure $\mu$ on $K$.

Proposition 3.6. Let $K$ be a compact Hausdorff topological space and $\mu$ a Radon measure on $K$. If $\iota(\mu)$ is Baire $\left(C(K), \mathfrak{T}_{p}(K)\right)$-measurable, then there is a closed separable set $F \subset K$ such that $\mu(K \backslash F)=0$.

Proof. In view of Lemma 3.5, there is a countable set $D \subset K$ such that

$$
\iota(\mu) \in \overline{\operatorname{span}\left\{\delta_{t}: t \in D\right\}}{ }^{\text {weak }^{*}} .
$$

Define $F:=\bar{D}$ and fix $t \in K \backslash F$. By Urysohn's lemma, there is a continuous function $f: K \rightarrow[0,1]$ such that $f(t)=1$ and $f(s)=0$ for every $s \in F$. Given $\varepsilon>0$, there exist $t_{1}, \ldots, t_{n} \in D$ and $a_{1}, \ldots, a_{n} \in \mathbb{R}$ such that

$$
\int_{K} f d \mu=\left|\int_{K} f d \mu-\sum_{i=1}^{n} a_{i} f\left(t_{i}\right)\right|=\left|\langle\iota(\mu), f\rangle-\left\langle\sum_{i=1}^{n} a_{i} \delta_{t_{i}}, f\right\rangle\right| \leq \varepsilon .
$$

As $\varepsilon>0$ is arbitrary, the open set $G_{t}:=\{s \in K: f(s)>0\}$ satisfies $\mu\left(G_{t}\right)=0$. Since $K \backslash F=\bigcup\left\{G_{t}: t \in K \backslash F\right\}$ and $\mu$ is a Radon measure, we conclude that $\mu(K \backslash F)=0$. This completes the proof. that

COROLlary 3.7. Let $K$ be a compact Hausdorff topological space such

$$
\operatorname{Baire}\left(C(K), \mathfrak{T}_{p}(K)\right)=\operatorname{Baire}(C(K), w) .
$$

Then for each Radon measure $\mu$ on $K$ there is a closed separable set $F \subset K$ such that $\mu(K \backslash F)=0$.

EXAMPLE 3.8. Under the continuum hypothesis, there exists a compact Hausdorff topological space $K$ (the so-called Kunen-Haydon-Talagrand space) with the following properties (see [19, §5]): (i) $K$ is first-countable; (ii) $K$ is not separable; (iii) there is a Radon measure $\mu$ on $K$ such that $\mu(G)>0$ for every non-empty open set $G \subset K$. On the one hand, (i) implies that $C(K)$ has the PIP (see [22, Theorem 3]). On the other hand, in view of (ii) and (iii), an appeal to Corollary 3.7 establishes that Baire $\left(C(K), \mathfrak{T}_{p}(K)\right)$ $\neq \operatorname{Baire}(C(K), w)$.

A well known theorem due to Odell and Rosenthal [20] (cf. [5, Theorem 4.1]) states that a separable Banach space $Y$ is weak ${ }^{*}$-sequentially dense in $Y^{* *}$ if and only if $Y \not \supset \ell^{1}$. In Proposition 3.9 below we apply this result to analyze the coincidence of $\operatorname{Baire}\left(Y^{*}, w^{*}\right)$ and $\operatorname{Baire}\left(Y^{*}, w\right)$ for a not necessarily separable Banach space $Y$. Recall first (Haydon [16], cf. [5, Theorem 6.8]) that $Y \not \supset \ell^{1}$ if and only if each $y^{* *} \in Y^{* *}$ is $\operatorname{Univ}\left(Y^{*}, w^{*}\right)$-measurable, that is, if and only if $\operatorname{Baire}\left(Y^{*}, w\right) \subset \operatorname{Univ}\left(Y^{*}, w^{*}\right)$.

Proposition 3.9. Let $Y$ be a Banach space. The following conditions are equivalent: 
(i) $Y$ is weak $k^{*}$-sequentially dense in $Y^{* *}$;

(ii) $\operatorname{Baire}\left(Y^{*}, w^{*}\right)=\operatorname{Baire}\left(Y^{*}, w\right)$;

(iii) $Y \not \supset \ell^{1}$ and for each $y^{* *} \in Y^{* *}$ there is a countable set $D \subset Y$ such that $y^{* *} \in \bar{D}^{\text {weak* }}$.

Proof. (i) $\Rightarrow$ (ii) is obvious. Let us turn to the proof of (ii) $\Rightarrow$ (iii). Since

$$
\operatorname{Baire}\left(Y^{*}, w\right)=\operatorname{Baire}\left(Y^{*}, w^{*}\right) \subset \operatorname{Borel}\left(Y^{*}, w^{*}\right) \subset \operatorname{Univ}\left(Y^{*}, w^{*}\right),
$$

the aforementioned Haydon's result ensures that $Y \not \supset \ell^{1}$. On the other hand, given $y^{* *} \in Y^{* *}$, Lemma 3.5 can be applied to find a countable set $C \subset Y$ such that $y^{* *} \in \overline{\operatorname{span}(C)}^{\text {weak* }^{*}}$. Clearly, the set $D \subset Y$ of all linear combinations of elements of $C$ with rational coefficients is countable and $y^{* *} \in \bar{D}^{\text {weak* }^{*}}$. This establishes (ii) $\Rightarrow$ (iii).

The proof of (iii) $\Rightarrow$ (i) is as follows. Fix $y^{* *} \in Y^{* *}$. Take a countable set $D \subset Y$ such that $y^{* *} \in \bar{D}^{\text {weak }^{*}}$ and define $Z:=\overline{\operatorname{span}(D)}\|\cdot\|$. Since

$$
\left\{y^{*} \in Y^{*}: y^{*}(z)=0 \text { for every } z \in Z\right\}=\bigcap_{y \in D} \operatorname{ker} y \subset \operatorname{ker} y^{* *},
$$

there exists $z^{* *} \in Z^{* *}$ such that $y^{* *}=z^{* *} \circ r$, where $r: Y^{*} \rightarrow Z^{*}$ is the "restriction" operator. Notice that $Z$ is separable and $Z \not \supset \ell^{1}$, so the OdellRosenthal theorem allows us to obtain a sequence $\left(z_{n}\right)$ in $Z \subset Y$ that converges to $z^{* *}$ in $\left(Z^{* *}\right.$, weak $\left.{ }^{*}\right)$. Clearly, $\left(z_{n}\right)$ converges to $y^{* *}$ in $\left(Y^{* *}\right.$, weak $\left.{ }^{*}\right)$. The proof is complete.

The previous proposition can be applied to the class of dual Banach spaces with property $(\mathrm{C})$. To this end, we use the following characterization due to Pol [23]: a Banach space $X$ has property (C) if and only if for every $A \subset B_{X^{*}}$ and every $x^{*} \in \bar{A}^{\text {weak }^{*}}$ there is a countable set $E \subset A$ such that $x^{*} \in \overline{\mathrm{co}(E)}^{\text {weak }^{*} \text {. }}$.

Corollary 3.10. Let $Y$ be a Banach space such that $Y^{*}$ has property (C). Then

$$
\operatorname{Baire}\left(Y^{*}, w^{*}\right)=\operatorname{Baire}\left(Y^{*}, w\right) \text {. }
$$

Proof. We will check that $Y$ meets the requirements of Proposition 3.9(iii). On the one hand, since $\ell_{\infty}$ fails property (C) and this property is clearly inherited by quotients, we infer that $Y \not \supset \ell^{1}$. On the other hand, given $y^{* *} \in$ $B_{Y^{* *}}=\bar{B}_{Y}{ }^{\text {weak }^{*}}$, the previous result of Pol says that there is a countable set $E \subset B_{Y}$ such that $y^{* *} \in \overline{\operatorname{co}(E)}^{\text {weak }^{*}}$, and so the set $D$ of all convex combinations of elements of $E$ with rational coefficients is countable and $y^{* *} \in \bar{D}^{\text {weak }^{*}}$.

REMARK 3.11. The converse of the previous corollary holds true for $Y$ separable. Indeed, if $\operatorname{Baire}\left(Y^{*}, w^{*}\right)=\operatorname{Baire}\left(Y^{*}, w\right)$, then $Y$ is weak*- 
sequentially dense in $Y^{* *}$. Thus $\left(B_{Y^{* *}}\right.$, weak $\left.{ }^{*}\right)$ can be thought of as a subspace of the space $\left(B_{1}\left(B_{Y^{*}}, w^{*}\right), \mathfrak{T}_{p}\left(B_{Y^{*}}\right)\right)$ of all real-valued Baire-1 functions on $\left(B_{Y^{*}}, w^{*}\right)$, which is angelic since $\left(B_{Y^{*}}, w^{*}\right)$ is a Polish space (Bourgain, Fremlin and Talagrand [2], cf. [5, Theorem 4.1]). Hence $\left(B_{Y^{* *}}\right.$, weak $\left.^{*}\right)$ is angelic as well and so $Y^{*}$ has property (C).

Recall that a cardinal $\kappa$ is of measure zero (or measure-free) if there is no probability measure $\mu$ on the power set of $\kappa$ such that $\mu(\{\alpha\})=0$ for every $\alpha<\kappa$. A well known theorem of Ulam (see e.g. [13, 438C]) asserts that the first uncountable ordinal, denoted by $\omega_{1}$, is of measure zero. We stress that it is consistent with ZFC to assume that every cardinal is of measure zero. For a detailed account on this subject we refer the reader to [13, §438] and the references therein.

EXAmPle 3.12. The space $\ell^{1}\left(\omega_{1}\right)=c_{0}\left(\omega_{1}\right)^{*}$ has the PIP and

$$
\operatorname{Baire}\left(\ell^{1}\left(\omega_{1}\right), w^{*}\right) \neq \operatorname{Baire}\left(\ell^{1}\left(\omega_{1}\right), w\right) .
$$

Proof. $\ell^{1}\left(\omega_{1}\right)$ has the PIP because $\omega_{1}$ is of measure zero (see [8, Theorem 5.10]). On the other hand, since $c_{0}\left(\omega_{1}\right)$ is not weak ${ }^{*}$-sequentially dense in its bidual $c_{0}\left(\omega_{1}\right)^{* *}=\ell_{\infty}\left(\omega_{1}\right)$, we can apply Proposition 3.9 to conclude that Baire $\left(\ell^{1}\left(\omega_{1}\right), w^{*}\right)$ and Baire $\left(\ell^{1}\left(\omega_{1}\right), w\right)$ are different.

In fact, we have Baire $\left(\ell^{1}\left(\omega_{1}\right), w\right)=\operatorname{Borel}\left(\ell^{1}\left(\omega_{1}\right),\|\cdot\|\right)$ (see [12]).

EXample 3.13 . The space $C[0,1]^{*}$ satisfies

$$
\operatorname{Baire}\left(C[0,1]^{*}, w^{*}\right) \neq \operatorname{Baire}\left(C[0,1]^{*}, w\right),
$$

and it has the PIP if and only if the cardinal of the continuum is of measure zero.

Proof. The last assertion was proved in [8]. On the other hand, since $\ell^{1}$ embeds in $C[0,1]$, we have $\operatorname{Baire}\left(C[0,1]^{*}, w^{*}\right) \neq \operatorname{Baire}\left(C[0,1]^{*}, w\right)$, by Proposition 3.9.

3.2. Uniqueness of extensions in dual Banach spaces. In this subsection we focus our attention on the class of dual Banach spaces $Y^{*}$ with the UMEP (in the sense of Definition 1.1), that is, those for which Baire $\left(Y^{*}, w^{*}\right)$ has the uniqueness property with respect to $\mathcal{M}_{\sigma}\left(Y^{*}, w\right)$. As an immediate consequence of Corollary 3.4 we get the following result.

Corollary 3.14. Let $Y$ be a Banach space. If $Y^{*}$ has the PIP, then $Y^{*}$ has the UMEP.

It turns out that the converse of the previous corollary holds whenever $Y \not \supset \ell^{1}$ (Theorem 3.17 below). Recall first that, for a Banach space $Y$ such that $Y \not \supset \ell^{1}$, we have

$$
\operatorname{Baire}\left(Y^{*}, w\right) \subset \operatorname{Univ}\left(Y^{*}, w^{*}\right),
$$


so the completion $\widetilde{\nu}$ of each Radon measure $\nu$ on $\operatorname{Borel}\left(Y^{*}, w^{*}\right)$ can be restricted to $\operatorname{Baire}\left(Y^{*}, w\right)$. We will use the notation $\nu^{0}=\left.\widetilde{\nu}\right|_{\operatorname{Baire}\left(Y^{*}, w\right)}$.

Proposition 3.15. Let $Y$ be a Banach space such that $Y \not \supset \ell^{1}$ and $\mu \in \mathcal{M}_{\sigma}\left(Y^{*}, w\right)$. The following conditions are equivalent:

(i) there is a Radon measure $\nu$ on $\operatorname{Borel}\left(Y^{*}, w^{*}\right)$ such that $\nu^{0}=\mu$;

(ii) $\mu$ is convexly $\tau$-additive.

Proof. For (i) $\Rightarrow\left(\right.$ ii), fix $n \in \mathbb{N}$ and consider the restriction $\nu_{n}$ of $\nu$ to

$$
\operatorname{Borel}\left(n B_{Y^{*}}, w^{*}\right)=\left\{B \cap n B_{Y^{*}}: B \in \operatorname{Borel}\left(Y^{*}, w^{*}\right)\right\} .
$$

Since $Y \not \supset \ell^{1}$, a result of Haydon [16] (cf. [5, Theorem 6.8]) ensures that the identity mapping $I_{n}: n B_{Y^{*}} \rightarrow Y^{*}$ is Pettis integrable with respect to the completion $\widetilde{\nu}_{n}$ of $\nu_{n}$. Therefore, its image measure $\mu_{n}:=\widetilde{\nu}_{n} I_{n}^{-1} \in \mathcal{M}_{\sigma}\left(Y^{*}, w\right)$ is convexly $\tau$-additive.

To prove that $\mu$ is convexly $\tau$-additive, consider a decreasing net $\left(C_{\alpha}\right)$ of convex closed elements of $\operatorname{Baire}\left(Y^{*}, w\right)$ with $\bigcap \mathcal{C}=\emptyset$. Fix $\varepsilon>0$ and take $n \in \mathbb{N}$ large enough such that $\nu\left(Y^{*} \backslash n B_{Y^{*}}\right) \leq \varepsilon / 2$. Since $\mu_{n}$ is convexly $\tau$-additive, we can find an $\alpha$ such that $\widetilde{\nu}_{n}\left(C_{\alpha} \cap n B_{Y^{*}}\right)=\mu_{n}\left(C_{\alpha}\right) \leq \varepsilon / 2$, hence

$$
\begin{aligned}
\mu\left(C_{\alpha}\right) & =\nu^{0}\left(C_{\alpha}\right)=\widetilde{\nu}\left(C_{\alpha} \cap n B_{Y^{*}}\right)+\widetilde{\nu}\left(C_{\alpha} \backslash n B_{Y^{*}}\right) \\
& =\widetilde{\nu}_{n}\left(C_{\alpha} \cap n B_{Y^{*}}\right)+\widetilde{\nu}\left(C_{\alpha} \backslash n B_{Y^{*}}\right) \leq \varepsilon .
\end{aligned}
$$

As $\varepsilon>0$ is arbitrary, $\lim _{\alpha} \mu\left(C_{\alpha}\right)=0$. This proves that $\mu$ is convexly $\tau$ additive.

Let us turn to the proof of (ii) $\Rightarrow(\mathrm{i})$. Since $\left(Y^{*}, w^{*}\right)$ is $\sigma$-compact (i.e. $Y^{*}$ can be expressed as a union of countably many $w^{*}$-compact subsets), the restriction $\left.\mu\right|_{\mathrm{Baire}\left(Y^{*}, w^{*}\right)}$ is tight and therefore it can be extended to a unique Radon measure $\nu$ on $\operatorname{Borel}\left(Y^{*}, w^{*}\right)$. Then both $\mu$ and $\nu^{0}$ are convexly $\tau$-additive (bear in mind the implication (i) $\Rightarrow($ ii) $)$ and $\left.\mu\right|_{\operatorname{Baire}\left(Y^{*}, w^{*}\right)}=$ $\left.\nu^{0}\right|_{\text {Baire }\left(Y^{*}, w^{*}\right)}$. An appeal to Theorem 3.2 now establishes that $\mu=\nu^{0}$, and the proof is complete.

The arguments of the proof of (ii) $\Rightarrow$ (i) in Proposition 3.15 also allow us to deduce the following corollary.

Corollary 3.16. Let $Y$ be a Banach space such that $Y \not \supset \ell^{1}$. Then every measure on Baire $\left(Y^{*}, w^{*}\right)$ can be extended in a unique way to a convexly $\tau$-additive measure on $\operatorname{Baire}\left(Y^{*}, w\right)$. If , in addition, $Y^{*}$ has the PIP, then every measure on $\operatorname{Baire}\left(Y^{*}, w^{*}\right)$ can be extended in a unique way to a measure on $\operatorname{Baire}\left(Y^{*}, w\right)$

Theorem 3.17. Let $Y$ be a Banach space such that $Y \not \supset \ell^{1}$. Then $Y^{*}$ has the PIP if and only if it has the UMEP. 
Proof. It only remains to prove the if part. To this end, we will show that each $\mu \in \mathcal{M}_{\sigma}\left(Y^{*}, w\right)$ is convexly $\tau$-additive. Indeed, as in the proof of the implication (ii) $\Rightarrow$ (i) in Proposition 3.15, we can find a Radon measure $\nu$ on $\operatorname{Borel}\left(Y^{*}, w^{*}\right)$ such that $\left.\mu\right|_{\operatorname{Baire}\left(Y^{*}, w^{*}\right)}=\left.\nu^{0}\right|_{\operatorname{Baire}\left(Y^{*}, w^{*}\right)}$. Since $Y^{*}$ has the UMEP, we get $\mu=\nu^{0}$ and an appeal to Proposition 3.15 establishes that $\mu$ is convexly $\tau$-additive, as required.

A completely regular Hausdorff topological space $(T, \mathfrak{T})$ is said to be realcompact if it is homeomorphic to a closed subset of $\mathbb{R}^{I}$ for some set $I$. A well known result of Hewitt and Shirota (see e.g. [27, Theorem 5, p. 218]) states that $(T, \mathfrak{T})$ is realcompact if and only if for each $\{0,1\}$-valued measure $\mu$ on Baire $(T, \mathfrak{T})$ there exists $t \in T$ such that $\mu(A)=1$ if $t \in A$ and $\mu(A)=0$ if $t \notin A$. This characterization was used in [8] (see [6] for a corrected proof) to show that every Banach space $X$ with the PIP is realcompact for its weak topology. We next obtain the same conclusion for any dual Banach space with the UMEP.

Proposition 3.18. Let $Y$ be a Banach space. If $Y^{*}$ has the UMEP, then $\left(Y^{*}, w\right)$ is realcompact.

Proof. Fix any $\mu \in \mathcal{M}_{\sigma}\left(Y^{*}, w\right)$ with $\mu\left(\operatorname{Baire}\left(Y^{*}, w\right)\right)=\{0,1\}$. Since $\left.\mu\right|_{\operatorname{Baire}\left(Y^{*}, w^{*}\right)}$ is tight and takes only the values 0 and 1 , it is not difficult to see that there exists $y_{0}^{*} \in Y^{*}$ such that for every $B \in \operatorname{Baire}\left(Y^{*}, w^{*}\right)$ we have $\mu(B)=1$ if $y_{0}^{*} \in B$ and $\mu(B)=0$ if $y_{0}^{*} \notin B$. Define $\mu^{\prime} \in \mathcal{M}_{\sigma}\left(Y^{*}, w\right)$ by

$$
\mu^{\prime}(A)=\left\{\begin{array}{ll}
1 & \text { if } y_{0}^{*} \in A \\
0 & \text { if } y_{0}^{*} \notin A
\end{array} \quad \text { for every } A \in \operatorname{Baire}\left(Y^{*}, w\right)\right.
$$

Since $\left.\mu\right|_{\operatorname{Baire}\left(Y^{*}, w^{*}\right)}=\left.\mu^{\prime}\right|_{\operatorname{Baire}\left(Y^{*}, w^{*}\right)}$ and $Y^{*}$ has the UMEP, we conclude that $\mu=\mu^{\prime}$. An appeal to the aforementioned characterization of Hewitt and Shirota establishes that $\left(Y^{*}, w\right)$ is realcompact.

The converse of the previous proposition does not hold in general. Indeed, the space $\ell_{\infty}=\left(\ell^{1}\right)^{*}$ is weakly realcompact (see [3, Example 1]) whereas it fails the UMEP, as we show in Example 3.19 below. This example was pointed out to us by D. H. Fremlin, who has kindly given his permission to include it here.

We first need to introduce the so-called Talagrand's measure [25] (see also e.g. [26, Section 13] or $[13, \S 464])$. We identify $\{0,1\}^{\mathbb{N}}$ with $\mathcal{P}(\mathbb{N})$ by means of the bijection $\zeta:\{0,1\}^{\mathbb{N}} \rightarrow \mathcal{P}(\mathbb{N})$ defined by $\zeta\left(\left(a_{n}\right)\right):=\left\{n \in \mathbb{N}: a_{n}=1\right\}$. Let $\left(\{0,1\}^{\mathbb{N}}, \Sigma, \lambda\right)$ be the complete probability space obtained after completing the usual product probability measure on $\{0,1\}^{\mathbb{N}}$. Recall that Talagrand's measure, which we denote by $\lambda_{1}$, is a complete extension of $\lambda$ to a larger $\sigma$ algebra on $\{0,1\}^{\mathbb{N}}$, say $\Sigma_{1} \supset \Sigma$, such that for every free ultrafilter $\mathcal{U} \subset \mathcal{P}(\mathbb{N})$ we have $\zeta^{-1}(\mathcal{U}) \in \Sigma_{1}$ and $\lambda_{1}\left(\zeta^{-1}(\mathcal{U})\right)=1$. 


\section{EXAMPLE 3.19. $\ell_{\infty}$ does not have the UMEP.}

Proof. Fremlin and Talagrand [14] (cf. [26, 13-3-3]) showed that the identity mapping $f:\{0,1\}^{\mathbb{N}} \rightarrow \ell_{\infty}$ is scalarly measurable with respect to $\lambda_{1}$. In fact, they proved that for every $y^{* *} \in \ell_{\infty}^{*}$ there exist $y \in \ell^{1}$ and $\alpha \in \mathbb{R}$ such that $\left\langle y^{* *}, f\right\rangle=\langle f, y\rangle+\alpha \lambda_{1}$-a.e. Therefore, we can consider the induced image measure $\mu_{1}:=\lambda_{1} f^{-1} \in \mathcal{M}_{\sigma}\left(\ell_{\infty}, w\right)$.

Let $\phi:\{0,1\}^{\mathbb{N}} \rightarrow\{0,1\}^{\mathbb{N}}$ be the bijection given by $\phi(a):=\zeta^{-1}(\mathbb{N} \backslash \zeta(a))$. Then for every $B \in \Sigma$ we have $\phi(B) \in \Sigma$ and $\lambda(\phi(B))=\lambda(B)$. Moreover, it was shown in [25] that $\Sigma_{1}=\left\{\phi(B): B \in \Sigma_{1}\right\}$. Clearly, the function $\lambda_{2}$ : $\Sigma_{1} \rightarrow[0,1]$ given by $\lambda_{2}(A):=\lambda_{1}(\phi(A))$ is a complete measure extending $\lambda$.

We claim that $f$ is scalarly measurable with respect to $\lambda_{2}$. Indeed, given $y^{* *} \in \ell_{\infty}^{*}$, we already know that there exist $y \in \ell^{1}, \alpha \in \mathbb{R}$ and $B \in \Sigma_{1}$ with $\lambda_{1}(B)=1$ such that $\left\langle y^{* *}, f(b)\right\rangle=\langle y, f(b)\rangle+\alpha$ for every $b \in B$. A simple computation yields

$$
\left\langle y^{* *}, f(a)\right\rangle=\left\langle y^{* *}, \mathbf{1}\right\rangle-\langle y, \mathbf{1}\rangle+\langle y, f(a)\rangle-\alpha \quad \text { for every } a \in \phi(B),
$$

where $\mathbf{1}=(1,1, \ldots) \in \ell_{\infty}$. Since $\lambda_{2}(\phi(B))=1$ and $\langle y, f\rangle$ is $\Sigma$-measurable (notice that $f$ is $w^{*}$-continuous), we infer that $\left\langle y^{* *}, f\right\rangle$ is measurable with respect to $\lambda_{2}$, as claimed. So we can take the induced image measure $\mu_{2}:=$ $\lambda_{2} f^{-1} \in \mathcal{M}_{\sigma}\left(\ell_{\infty}, w\right)$.

On the one hand, since $f^{-1}(B) \in \Sigma$ for every $B \in \operatorname{Baire}\left(\ell_{\infty}, w^{*}\right)$ and $\left.\lambda_{1}\right|_{\Sigma}=\lambda=\left.\lambda_{2}\right|_{\Sigma}$, we conclude that $\left.\mu_{1}\right|_{\operatorname{Baire}\left(\ell_{\infty}, w^{*}\right)}=\left.\mu_{2}\right|_{\operatorname{Baire}\left(\ell_{\infty}, w^{*}\right)}$.

On the other hand, fix a free ultrafilter $\mathcal{U} \subset \mathcal{P}(\mathbb{N})$ and consider its associated functional $x_{\mathcal{U}}^{* *} \in \ell_{\infty}^{*}$ given by $x_{\mathcal{U}}^{* *}\left(\left(a_{n}\right)\right)=\lim _{n \rightarrow \mathcal{U}} a_{n}$. It is clear that

$$
H:=\left\{a \in \ell_{\infty}: x_{\mathcal{U}}^{* *}(a)=1\right\} \in \operatorname{Baire}\left(\ell_{\infty}, w\right)
$$

satisfies $f^{-1}(H)=\zeta^{-1}(\mathcal{U})$, so $\mu_{1}(H)=\lambda_{1}\left(\zeta^{-1}(\mathcal{U})\right)=1$, whereas

$$
\begin{aligned}
\mu_{2}(H) & =\lambda_{2}\left(\zeta^{-1}(\mathcal{U})\right) \\
& =\lambda_{1}\left(\left\{a \in\{0,1\}^{\mathbb{N}}: \phi(a) \in \zeta^{-1}(\mathcal{U})\right\}\right)=\lambda_{1}\left(\{0,1\}^{\mathbb{N}} \backslash \zeta^{-1}(\mathcal{U})\right)=0 .
\end{aligned}
$$

Therefore, $\mu_{1} \neq \mu_{2}$. It follows that $\ell_{\infty}$ does not have the UMEP.

We finish the paper with some further comments about the PIP and the UMEP in a dual Banach space $Y^{*}$.

Bator asked in [1] whether a scalarly bounded function $f$ defined on a measure space $(\Lambda, \mathcal{S}, \nu)$ with values in $Y^{*}$ is Pettis integrable provided that the following condition (necessary for Pettis integrability) holds:

$(* *) \quad$ for each $y^{* *} \in Y^{* *}$ there exists a bounded sequence $\left(y_{n}\right)$ in $Y$ such that $\lim _{n}\left\langle y_{n}, f\right\rangle=\left\langle y^{* *}, f\right\rangle \nu$-a.e.

Musiał and Plebanek [18] (assuming the existence of a two-valued measurable cardinal) and Stefánsson [24] (without additional set-theoretic assumptions) gave examples showing that the answer to Bator's question is negative in 
general. However, $Y^{*}$ has the $\nu$-PIP if and only if every scalarly bounded function $f: \Lambda \rightarrow Y^{*}$ satisfies (**) (see [18]).

Next let us translate these facts into the language of measures on $\operatorname{Baire}\left(Y^{*}, w\right)$. Observe first that condition $(* *)$ above is equivalent to saying that the image measure $\nu f^{-1}$ on $\operatorname{Baire}\left(Y^{*}, w\right)$ has the following property:

Definition 3.20. Let $Y$ be a Banach space and $\mu \in \mathcal{M}_{\sigma}\left(Y^{*}, w\right)$. We say that $\mu$ has the Bator property if for each $y^{* *} \in Y^{* *}$ there exists a bounded sequence $\left(y_{n}\right)$ in $Y$ converging to $y^{* *} \mu$-a.e.

Every convexly $\tau$-additive measure on $\operatorname{Baire}\left(Y^{*}, w\right)$ has the Bator property (see the proof of Theorem 2.7), but the converse does not hold in general (consider the image measures of the functions constructed in [18] and [24]). According to the results above, we can now state that $Y^{*}$ has the PIP if and only if every measure on $\operatorname{Baire}\left(Y^{*}, w\right)$ has the Bator property.

Note that every measure on $\operatorname{Baire}\left(Y^{*}, w\right)$ with the Bator property is approximated by Baire $\left(Y^{*}, w^{*}\right)$ (apply Lemma 2.4). Bearing this in mind, the previous characterization of the PIP should now be compared with the following straightforward consequence of Corollary 2.3:

Corollary 3.21. Let $Y$ be a Banach space. Then $Y^{*}$ has the UMEP if and only if every measure on $\operatorname{Baire}\left(Y^{*}, w\right)$ is approximated by $\operatorname{Baire}\left(Y^{*}, w^{*}\right)$.

One question still unanswered is whether the PIP and the UMEP are equivalent for arbitrary dual Banach spaces.

Acknowledgements. The authors are grateful to David Fremlin and Matías Raja for valuable discussions on the subject of this paper.

\section{References}

[1] E. M. Bator, Pettis integrability and the equality of the norms of the weak* integral and the Dunford integral, Proc. Amer. Math. Soc. 95 (1985), 265-270.

[2] J. Bourgain, D. H. Fremlin, and M. Talagrand, Pointwise compact sets of Bairemeasurable functions, Amer. J. Math. 100 (1978), 845-886.

[3] H. H. Corson, The weak topology of a Banach space, Trans. Amer. Math. Soc. 101 (1961), 1-15.

[4] R. G. Douglas, On extremal measures and subspace density, Michigan Math. J. 11 (1964), 243-246.

[5] D. van Dulst, Characterizations of Banach spaces not containing $l^{1}$, CWI Tract 59, Centrum voor Wiskunde en Informatica, Amsterdam, 1989.

[6] G. A. Edgar, Errata to "Measurability in a Banach space. II", available at URL http://www.math.ohio-state.edu/ edgar/publications/m2errata.ps.

[7] -, Measurability in a Banach space, Indiana Univ. Math. J. 26 (1977), 663-677.

[8] -, Measurability in a Banach space. II, ibid. 28 (1979), 559-579.

[9] -, On pointwise-compact sets of measurable functions, in: Measure Theory (Oberwolfach, 1981), Lecture Notes in Math. 945, Springer, Berlin, 1982, 24-28. 
[10] G. A. Edgar and L. Sucheston, Stopping Times and Directed Processes, Encyclopedia Math. Appl. 47, Cambridge Univ. Press, Cambridge, 1992.

[11] M. Fabian, Gâteaux Differentiability of Convex Functions and Topology. Weak Asplund Spaces, CMS Ser. Monogr. Adv. Texts, Wiley, New York, 1997.

[12] D. H. Fremlin, Borel sets in nonseparable Banach spaces, Hokkaido Math. J. 9 (1980), 179-183.

[13] -, Measure Theory. Vol. 4: Topological Measure Spaces, Torres Fremlin, Colchester, 2003.

[14] D. H. Fremlin and M. Talagrand, A decomposition theorem for additive set-functions, with applications to Pettis integrals and ergodic means, Math. Z. 168 (1979), $117-142$.

[15] A. B. Gulisashvili, Estimates for the Pettis integral in interpolation spaces, and a generalization of some imbedding theorems, Soviet Math. Dokl. 25 (1982), 428-432; transl. of: Dokl. Akad. Nauk SSSR 263 (1982), 793-798 (in Russian).

[16] R. Haydon, Some more characterizations of Banach spaces containing $l_{1}$, Math. Proc. Cambridge Philos. Soc. 80 (1976), 269-276.

[17] A. Ionescu Tulcea, On pointwise convergence, compactness, and equicontinuity. II, Adv. Math. 12 (1974), 171-177.

[18] K. Musial and G. Plebanek, Pettis integrability and the equality of the norms of the weak* integral and the Dunford integral, Hiroshima Math. J. 19 (1989), 329-332.

[19] S. Negrepontis, Banach spaces and topology, in: Handbook of Set-Theoretic Topology, North-Holland, Amsterdam, 1984, 1045-1142.

[20] E. Odell and H. P. Rosenthal, A double-dual characterization of separable Banach spaces containing $l^{1}$, Israel J. Math. 20 (1975), 375-384.

[21] D. Plachky, Extremal and monogenic additive set functions, Proc. Amer. Math. Soc. 54 (1976), 193-196.

[22] G. Plebanek, On Pettis integrals with separable range, Colloq. Math. 64 (1993), 71-78.

[23] R. Pol, On a question of H. H. Corson and some related problems, Fund. Math. 109 (1980), 143-154.

[24] G. F. Stefánsson, The $\mu-P I P$ and integrability of a single function, Proc. Amer. Math. Soc. 124 (1996), 539-542.

[25] M. Talagrand, Compacts de fonctions mesurables et filtres non mesurables, Studia Math. 67 (1980), 13-43.

[26] -, Pettis integral and measure theory, Mem. Amer. Math. Soc. 51 (1984), no. 307.

[27] V. S. Varadarajan, Measures on topological spaces, Amer. Math. Soc. Transl. (2) 48 (1965), 161-228; transl. of: Mat. Sb. (N.S.) 55 (97) (1961), 35-100.

[28] G. Vera, Pointwise compactness and continuity of the integral, Rev. Mat. Univ. Complut. Madrid 9 (1996), Special Issue, suppl., 221-245.

Departamento de Matemáticas

Universidad de Murcia

30100 Espinardo (Murcia), Spain

E-mail: joserr@um.es

gvb@um.es

Received May 10, 2005

Revised version March 16, 2006 